9th A. Friedmann International Seminar and

3rd Casimir Symposium 2015

International Journal of Modern Physics: Conference Series

Vol. 41 (2016) 1660137 (10 pages)

(C) The Author(s)

DOI: $10.1142 /$ S201019451660137X

\title{
Transport of laser emission with broadband spectrum in optically dense atomic medium under the coherent population trapping
}

\author{
K. A. Barantsev \\ Dept. of Quantum Electronics, Peter the Great St.Petersburg Polytechnic University, \\ Polytechnicheskaya 29, Saint Petersburg, 195251, Russia \\ kostmann@yandex.ru \\ A. N. Litvinov \\ Dept. of Quantum Electronics, Peter the Great St.Petersburg Polytechnic University, \\ Polytechnicheskaya 29, Saint Petersburg, 195251, Russia \\ andrey.litvinov@mail.ru \\ E. N. Popov \\ Dept. of Quantum Electronics, Peter the Great St.Petersburg Polytechnic University, \\ Polytechnicheskaya 29, Saint Petersburg, 195251, Russia \\ enp-tion@ya.ru
}

Received 17 September 2015

Published 18 March 2016

\begin{abstract}
The paper presents a theory describing the formation of the coherent population trapping resonance for the finite laser bandwidth in an optically-dense medium of atoms inside a buffer gas cell. The equations of the atomic density matrix are established, as well as the transfer equations of laser emission spectrum inside a cell with working and buffer gas at a determined temperature. The dependence of the quality of the coherent population trapping resonance on the laser bandwidth has been studied in the case of detecting the signal of the transmitted emission and the fluorescence signal.
\end{abstract}

Keywords: Coherent population trapping; optically dense medium; laser spectrum.

PACS numbers: 03.65.-w, 03.50.De

\section{Introduction}

The interaction of multi-frequency laser emission with quantum systems can lead to some new nonlinear optic effects. Among those effects one can mention the coherent population trapping effect $(\mathrm{CPT})^{1}$ and the related electromagnetically-induced

This is an Open Access article published by World Scientific Publishing Company. It is distributed under the terms of the Creative Commons Attribution 4.0 (CC-BY) License. Further distribution of this work is permitted, provided the original work is properly cited. 
transparency effect (EIT). ${ }^{2,3}$ The CPT effect for a simple non-degenerate $\Lambda$-scheme lies in the emergence of quantum interference between the excitation channels of the two-frequency laser emission. This leads to the absence of absorption in a certain frequency domain in the given medium, and, as a result, there appears a spectral window. The width of the spectral window at fairly low laser emission intensities is defined by the rate of the radio-frequency coherence disintegration, which is five exponential parts lower in alkali atoms than the rate of disintegration of optical coherences. This peculiarity helps to use this spectral window as a basis for the development of quantum frequency standards, ${ }^{4}$ optical magnetometers, ${ }^{5,6}$ highresolution spectroscopic tools, ${ }^{7,8}$ tools to record and store quantum information, 9,10 etc.

The CPT in cells is discussed in a substantial number of papers. One of the directions of research is studying the shape of the CPT resonance on the basis of rubidium atoms in a buffer gas cell for continuous emission. Thus, Ref. 11 provides a theoretical basis for the possibility of pseudo-resonance in the field of co-directional waves. These results are confirmed in the course of experiment in Ref. 12. These studies were further developed in Refs. 13, 14 focusing on miniature atomic clocks. A theoretical and experimental research of optical pumping of cesium atoms in laser fields directed perpendicularly, as well as the CPT effect in those atoms is presented in Ref 15 . The theory of the Dicke effect for the CPT resonance in a buffer gas cell is built in Ref. 16 .

The research conducted earlier (mainly theoretical) was aimed at determining the influence of particular factors on the CPT resonance shape. At the same time it should be mentioned that at a certain temperature necessary to reach the requested concentration of the active atoms the medium (consisting of those atoms) becomes optically dense, which leads to the absorption of emission in the cell. Not less important is the fact that laser emission is not monochromatic, but it possesses a finite emission bandwidth. Different regions of the spectrum can be absorbed irregularly, which leads to its distortion. A distorted spectrum causes changes in the conditions of CPT resonance excitation, which, as a result, influences the output signal and the resonance shape to be detected. Besides, buffer gas inside the cell with active atoms is vital for the Dicke effect ${ }^{17}$ to take place, as well as for a quite narrow CPT resonance to be observed. A consistent theory describing all those factors has not been built so far, there have only been some attempts to build a theory of the CPT effect when using non-monochromatic lamp pumping in an optically-dense medium. ${ }^{18}$ Thus, the present paper is aimed at building a theoretical model describing the interaction between the optically-dense medium of three-level $\Lambda$-atoms and bi-chromatic laser emission possessing a finite laser bandwidth.

\section{Problem Statement and Theory}

Let us consider the interaction between two-frequency laser emission and the saturated vapor of $\mathrm{Rb}$ atoms (active atoms) inside a gas cell at temperatures of about 
a)

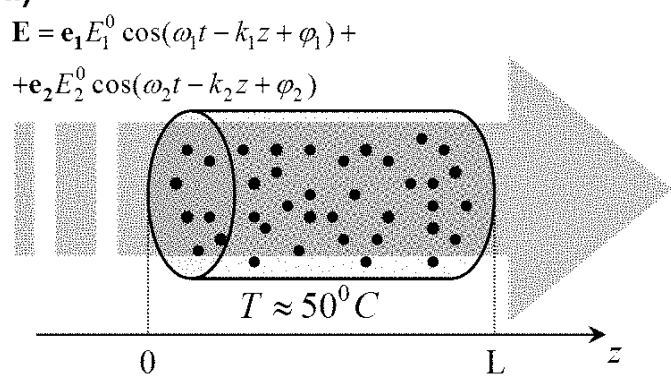

b)

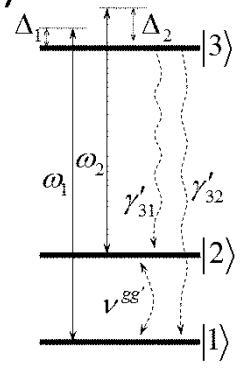

Fig. 1. (a) Gas cell containing alkali atoms and buffer gas which is exposed to two-frequency laser emission. (b) The scheme of quantum levels of a $\mathrm{Rb}$ atom. $D_{1}$-line exposed to laser fields with frequencies $\omega_{1,2} . \Delta_{1,2}$ are one-photon detunings from atomic transitions, $\gamma_{m n}^{\prime}$ are the rates of disintegration of level $|3\rangle$ populations according to the corresponding channels, $\nu^{g g^{\prime}}$ is the speed of populations shuffle between the lower levels due to collisions.

$50^{0}$ (Fig. 1(a)). Apart from the Rb atoms, buffer gas $N_{2}$ has been introduced in the cell whose concentration exceeds the concentration of the alkali metal by six exponential parts. At the same time the atoms of the buffer gas do not interact with the laser emission which influences the Rb atoms. The purpose of introducing the buffer gas lies in that in such surroundings the working atoms demonstrate diffusion and the mean free pass decreases dramatically. This substantially reduces the frequency at which the active atoms collide with the cell walls, which leads to an increase in the time of the coherent interaction with the laser field (the Dicke effect ${ }^{17}$ ), as well as a narrowing of the $\mathrm{CPT}$ resonance. ${ }^{19,20}$

Let us consider a laser field as a classical two-frequency plane wave with distribution along axis $z$. The electric field vector of the wave is as follows:

$$
\mathbf{E}(z, t)=\mathbf{e}_{\mathbf{1}} E_{1}^{0}(z, t) e^{-i\left[\omega_{1} t-k_{1} z+\varphi_{1}(t)\right]}+\mathbf{e}_{\mathbf{2}} E_{2}^{0}(z, t) e^{-i\left[\omega_{2} t-k_{2} z+\varphi_{2}(t)\right]}+\text { c.c. },
$$

where $\mathbf{e}_{\mathbf{j}}$ are unit polarization vectors, $k_{j}$ are wave numbers, $j=1,2$. Field amplitudes $E_{j}^{0}(z, t)$ and phases $\varphi_{j}(t)$ depend on time only due to fluctuations. Laser fields are considered to be stationary in the broad sense, that is the average values of $E_{j}^{0}(z), \varphi_{j}$ do not change when the averaging interval $\Delta t$ is shifted according to the time scale. The non-monochromatic nature of laser emission connected with laser noise leads to broadening of spectral lines. The width and the shape of lines are determined by the type of the noise.

The energy structure of a $\mathrm{Rb}$ atom $D_{1}$-line can be described with the help of a three-level model, in which the two lower levels $|1\rangle$ and $|2\rangle$ correspond to the hyperfine splitting of state ${ }^{2} S_{1 / 2}$, and the top level $|3\rangle$ corresponds to state ${ }^{2} P_{1 / 2}$ (Fig. 1(b)). The frequency component of the laser field $\omega_{1}$ is quasi-resonant to the transition $|1\rangle \leftrightarrow|3\rangle$, and $\omega_{2}$ - to the transition $|2\rangle \leftrightarrow|3\rangle$ (the wavelength equals 795 $\mathrm{nm})$. We suppose that the width of laser lines $\Gamma_{\text {las }}$ is significantly smaller than the value of the hyperfine splitting $\omega_{h f s}$ between the levels $|1\rangle$ and $|2\rangle$, which equals 6.8 
$\mathrm{GHz}$ in a ${ }^{87} \mathrm{Rb}$ atom. This helps to avoid the cross-coupling of frequency component $\omega_{1}$ in transition $|2\rangle \leftrightarrow|3\rangle$, and frequency component $\omega_{2}$ in transition $|1\rangle \leftrightarrow|3\rangle$.

Let us describe the condition of an atom with the help of a density matrix $\tilde{f}_{m n}(\mathbf{v}, \mathbf{r}, t)$ where $\mathbf{v}, \mathbf{r}$ is the velocity and the coordinate of an atom in the laboratory coordinate system. The quantum kinetic equations for the density matrix are as follows: ${ }^{21}$

$$
\begin{aligned}
\left(\frac{\partial}{\partial t}\right. & +\mathbf{v} \nabla) \tilde{f}_{m n}(\mathbf{v}, \mathbf{r}, t) \\
& =-\frac{i}{\hbar} \sum_{j=1}^{3}\left[H_{m j} \tilde{f}_{j n}(\mathbf{v}, \mathbf{r}, t)-\tilde{f}_{m j}(\mathbf{v}, \mathbf{r}, t) H_{j n}\right]+R_{m n}+S_{m n},
\end{aligned}
$$

where $\hat{H}$ is the Hamiltonian, $R$ is the relaxation matrix and $S$ is the collision integral. $m, n=1,2,3$. According to dipole approximation the Hamiltonian can be presented as:

$$
\begin{aligned}
\hat{H}= & \sum_{j=1}^{3} \varepsilon_{j}|j\rangle\left\langle j\left|-\hat{\mathbf{d}} \mathbf{E}=\sum_{j=1}^{3} \varepsilon_{j}\right| j\right\rangle\langle j| \\
& +\hbar \Omega_{1}(z, t) e^{-i\left[\omega_{1} t-k_{1} z\right]}|3\rangle\left\langle 1\left|+\hbar \Omega_{2}(z, t) e^{-i\left[\omega_{2} t-k_{2} z\right]}\right| 3\right\rangle\langle 2|+\text { h.c. },
\end{aligned}
$$

where $\Omega_{j}(z, t)=-d_{3 j} E_{j}^{0}(z, t) e^{-i \varphi_{j}(t)} / \hbar$ are the Rabi frequencies, which contain the amplitude and phase fluctuations, $\hat{\mathbf{d}}$ is the dipole momentum operator, $d_{3 j}$ are its matrix elements, $\varepsilon_{j}$ are the energies of atomic levels, $j=1,2$. The Rabi frequencies are random complex-valued functions of time. It is clear that the elements $\tilde{f}_{m n}(\mathbf{v}, \mathbf{r}, t)$ of the density matrix will be also random functions of time.

From the non-diagonal elements of the density matrix let us derive the factor $\tilde{f}_{m n}(v, z, t)=f_{m n}(v, z, t) e^{i\left(\omega_{j} t-k_{j} z\right)},(j=1,2, m, n=1,2,3)$ oscillating with the laser frequency and use the rotating wave approximation. Here in one-dimensional approximation $f_{m n}(\mathbf{v}, \mathbf{r}, t)=f_{m n}\left(v_{z}, z, t\right)$, and it is presupposed $v_{z} \equiv v$. In order to solve the system of equations (2) for the atomic density matrix let us average it over time. The averaging time should be much longer than the characteristic fluctuation time of the laser field $\Delta t>>1 / \Gamma_{\text {las }}$. At the same time let us consider only the double-correlation functions disintegrating the higher-order correlators:

$$
\left\langle\Omega_{m}^{*}(t) \Omega_{n}\left(t^{\prime}\right) f_{i j}\left(v, t^{\prime \prime}\right)\right\rangle=\left\langle\Omega_{m}^{*}(t) \Omega_{n}\left(t^{\prime}\right)\right\rangle\left\langle f_{i j}\left(v, t^{\prime \prime}\right)\right\rangle=\left\langle\Omega_{m}^{*}(t) \Omega_{n}\left(t^{\prime}\right)\right\rangle \rho_{i j}(v) .
$$

Here argument $z$ is not mentioned for brevity. According to the Wiener-Khinchin theorem, the signal correlation function is an inverse Fourier transform of its power spectrum

$$
\left\langle\Omega_{m}^{*}(t) \Omega_{n}\left(t^{\prime}\right)\right\rangle=\int_{-\infty}^{\infty} J_{m n}(\omega) e^{i \omega\left(t-t^{\prime}\right)} d \omega
$$

where $J_{11}(\omega)$ and $J_{22}(\omega)$ are the energy spectra of the laser emission frequency components which are centered at zero. $J_{12}(\omega)$ is a complex value called cross-spectrum which reflects the correlation between the frequency components $\omega_{1}$ and $\omega_{2}$. 
When passing through a gas cell laser emission is absorbed at the resonance transitions of active atoms. The absorption may be not the same for the various spectral regions due to the different values of the refractive index of the medium for the different frequencies of emission. Consequently, the spectrum can become distorted inside the gas cell. In order to take this into account correctly it is necessary to establish the spectrum transfer equations. The transfer equations for Rabi frequencies are as follows: ${ }^{22}$

$$
\frac{\partial \Omega_{j}(t)}{\partial z}+\frac{1}{c} \frac{\partial \Omega_{j}(t)}{\partial t}=i q_{j} f_{j 3}(t)
$$

where $q_{j}=\left(2 \pi n_{a} d_{3 j}^{2} \omega_{j}\right) /(c \hbar), n_{a}$ is the concentration of the working atoms, $j=1,2$. Let us further multiply the equation $(6)$ by $\Omega_{1}^{*}(t)$, and the complex conjugate (6) by $\Omega_{1}\left(t^{\prime}\right)$, after which we average the equations over time and add them. The average from the derivative with respect to time can be set to zero owing to the symmetrical distribution of random complex field amplitudes related to their averages:

$$
\frac{\partial}{\partial z}\left\langle\Omega_{m}^{*}(t) \Omega_{n}\left(t^{\prime}\right)\right\rangle=i\left[q_{n}\left\langle\Omega_{m}^{*}(t) f_{n 3}\left(t^{\prime}\right)\right\rangle-q_{m}\left\langle\Omega_{n}\left(t^{\prime}\right) f_{3 m}(t)\right\rangle\right], m, n=1,2 .
$$

Let us use the Fourier transform for (7):

$$
\frac{\partial J_{m n}(\omega, z)}{\partial z}=i \int_{-\infty}^{\infty} d \tau e^{-i \omega \tau}\left[q_{n}\left\langle\Omega_{m}^{*}(t) f_{n 3}(t-\tau)\right\rangle-q_{m}\left\langle\Omega_{n}(t-\tau) f_{3 m}(t)\right\rangle\right] .
$$

Let us substitute $f_{n 3}(t)$ for the non-diagonal elements of the density matrix (atomic coherences) using the system (2) and let us disintegrate the triple correlators in an analogous way (4), after which we apply the Wiener-Khinchin theorem. As a result, we receive the spectral density transfer equations:

$$
\begin{aligned}
\frac{\partial J_{11}(\omega, z)}{\partial z}= & 2 q_{1}\left\{\rho_{11}(z) J_{11}(\omega, z) \operatorname{Re}\left[W\left(\Delta_{1}, \omega\right)\right]\right. \\
& \left.+\operatorname{Re}\left[\rho_{12}(z) J_{12}(\omega, z) W\left(\Delta_{1}, \omega\right)\right]\right\}, \\
\frac{\partial J_{22}(\omega, z)}{\partial z}= & 2 q_{2}\left\{\rho_{22}(z) J_{22}(\omega, z) \operatorname{Re}\left[W\left(\Delta_{2}, \omega\right)\right]\right. \\
& \left.+\operatorname{Re}\left[\rho_{21}(z) J_{21}(\omega, z) W\left(\Delta_{2}, \omega\right)\right]\right\}, \\
\frac{\partial J_{12}(\omega, z)}{\partial z}= & q_{2} W\left(\Delta_{2}, \omega\right)\left\{\rho_{22}(z) J_{12}(\omega, z)+\rho_{21}(z) J_{11}(\omega, z)\right\} \\
& +q_{1} W^{*}\left(\Delta_{1}, \omega\right)\left\{\rho_{11}(z) J_{12}(\omega, z)+\rho_{21}(z) J_{22}(\omega, z)\right\},
\end{aligned}
$$

where $\rho_{m n}(z)=\int\left\langle f_{m n}(v, z, t)\right\rangle d v$. The real part of functions

$$
W\left(\Delta_{n}, \omega\right)=-\frac{\sqrt{\pi}}{k_{n} v_{t}} w^{*}\left(\frac{\Delta_{n}-\omega}{k_{n} v_{t}}+i \frac{\Gamma_{n 3}^{\prime}}{k_{n} v_{t}}\right)
$$

has physical significance of an absorption line of a gas medium and manifests itself as a Voigt profile, and the imaginary part is presented as a dispersive profile. In (12) $w(x)=e^{-x^{2}}[1-\operatorname{erf}(x)]$ is the complex error function, $v_{t}$ is the most probable 
thermal velocity of atoms, $\Delta_{n}$ are one-photon detunings from the atomic transitions, $\Gamma_{n 3}^{\prime}$ are the rates of disintegration of atomic coherences modified due to collisions.

The absorption of spectral density is described with the help of equations (9-10), and the cross spectrum transfer equation (11) contains the information about phase incursions.

\section{Discussion of Results}

The system of equations for the average values of the density matrix enables monitoring the condition of working atoms for the time intervals that are longer than the averaging time $\Delta t$. In the present paper we consider a laser spectrum whose bandwidth is within the range $\gamma \leq \Gamma_{\text {las }} \leq \Delta_{D}$. Here $\Delta_{D}$ is Doppler broadening, $\gamma$ is the rate of disintegration of the top atomic level. Let us evaluate the lower border of the averaging time for $\Gamma_{\text {las }}=\gamma$. In this case the averaging time is $\Delta t>>10^{-7} s$. In quantum frequency standards short-term stability is normally calculated for the averaging interval of about $1 \mathrm{sec}$, which exceeds the lower evaluation of $\Delta t$ by several exponential parts. That is why the system of equations (2) solved together with the spectrum transfer equations (9)-(11) helps to evaluate the short-term stability of quantum frequency standard using the CPT effect.

Let us present the energy spectrum of the frequency components of a laser as a Lorentz contour when it enters the cell, which corresponds to the stationary fluctuations of carrier frequencies: ${ }^{23}$

$$
\begin{aligned}
& J_{11}(\omega, 0)=I_{1}(0) \frac{8 \pi d_{31}^{2}}{c \hbar^{2}} \frac{\Gamma_{\text {las }}}{2 \pi\left(\omega^{2}+\Gamma_{\text {las }}^{2} / 4\right)}, \\
& J_{22}(\omega, 0)=I_{2}(0) \frac{8 \pi d_{32}^{2}}{c \hbar^{2}} \frac{\Gamma_{\text {las }}}{2 \pi\left(\omega^{2}+\Gamma_{\text {las }}^{2} / 4\right)}, \\
& J_{12}(\omega, 0)=\sqrt{J_{11}(\omega, 0) J_{22}(\omega, 0)},
\end{aligned}
$$

where $I_{n}(z)=\frac{c \hbar^{2}}{8 \pi d_{3 n}^{2}} \int_{-\infty}^{\infty} J_{n n}(\omega, z) d \omega$ is the intensity of laser fields.

Figure 2(a) shows the dependence of the normalized emission intensity on the two-photon detuning $\delta=\left(\Delta_{1}-\Delta_{2}\right) / 2$ when leaving a gas cell. This dependence is a form of CPT resonance. At the same time one-photon detuning is considered to equal zero $\left(\Delta=\left(\Delta_{1}+\Delta_{2}\right) / 2=0\right)$. The curves in Fig. 2(a) correspond to the various laser fields bandwidths, but of the same integral intensity when entering the cell. When $\delta=0$ the transmitted intensity reaches its peak, as the working atoms are in a CPT condition and the medium becomes more transparent. ${ }^{1}$ On the tails of the curves the detuning is far from the CPT resonance and one can observe a usual exponential absorption of light by the medium. Let us introduce a non-dimensional parameter

$$
A=\frac{\Gamma_{l a s}}{\Delta_{D}+\gamma / 2+\nu},
$$



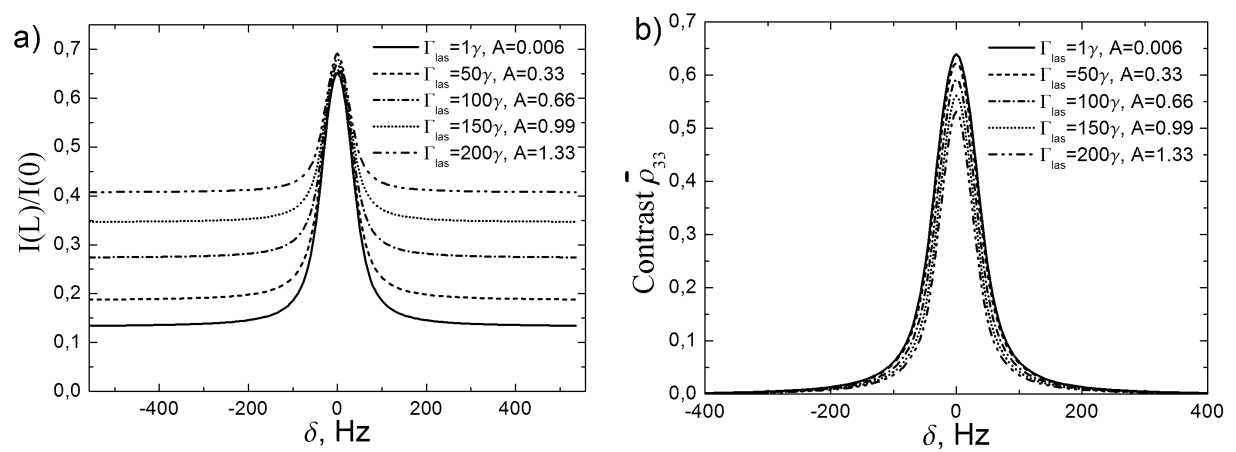

Fig. 2. The shape of the CPT resonance according to the emission intensity $I=I_{1}+I_{2}$ when leaving the cell (a), and according to the average population of the top level of working atoms (b) for various laser bandwidths. When the laser bandwidth changes, the integral emission intensity stays constant. Intensities when entering the cell $I_{1}(0)=I_{2}(0)=2 \mu \mathrm{W} / \mathrm{cm}^{2}$, temperature $T=$ $55^{0} \mathrm{C}$, the concentration of working atoms ${ }^{87} \mathrm{Rb} n_{a}=1.15 * 10^{11} \mathrm{~cm}^{-3}$, the concentration of buffer gas $N_{2} n_{b u f}=10^{18} \mathrm{~cm}^{-3}$, the length of cell $L=1 \mathrm{~cm}$.

which is the ratio of the laser bandwidth to the width of the working atoms absorption line. The latter is composed from Doppler broadening $\Delta_{D}$, natural width of the top level $\gamma / 2$ and collisional broadening $\nu$. When the temperature equals $50^{\circ} \mathrm{C}$ the Doppler and collisional broadening are comparable and exceed the homogeneous one by two exponential parts.

For $\Gamma_{l a s}=1 \gamma, 50 \gamma$ and $100 \gamma$ (the solid, dashed and dash-and-dot curves in Fig. 2(a)) the parameter $A<1$. It means that laser emission interacts with the atoms from a certain velocity group and not all ${ }^{87} \mathrm{Rb}$ atoms take part in the formation of the CPT resonance. Nevertheless, it is for these conditions that a stronger absorption is observed in comparison with the excitation conditions, when the bandwidth is $\Gamma_{\text {las }}>100 \gamma$ (the dotted curve and the dash-and-dot curve with two dots in Fig. 2(a)). It is explained by the fact that, according to the adiabatic approximation, the laser fields intensities are set as low. That is why the transitions $|1\rangle \leftrightarrow|3\rangle$ and $|2\rangle \leftrightarrow|3\rangle$ are not saturated and for each photon of the emission there will be a normal atom capable of absorbing it. This is performed for a wide range of $\Gamma_{l a s}$ change for which $A<<1$. At the same time the emission is absorbed homogeneously across the whole spectrum, and that is why it hardly becomes distorted (Fig. 3(a)). If $\Gamma_{l a s}$ is increased, parameter A becomes close to one. The number of photons on the edges of the spectrum becomes comparable or bigger than the number of atoms capable of absorbing them, that is why the output intensity outside the CPT resonance increases (the dotted curve in Fig. 2(a)).

For $\Gamma_{\text {las }}=200 \gamma$ parameter $A>1$. The photons possessing the frequencies in the domain separated from the central part of the spectrum interact with the fast atoms whose velocity is higher than the most probable thermal one. The number of these atoms is small in comparison with the number of photons, that is why such photons will be practically not absorbed. Besides, in the central part of the spectrum 

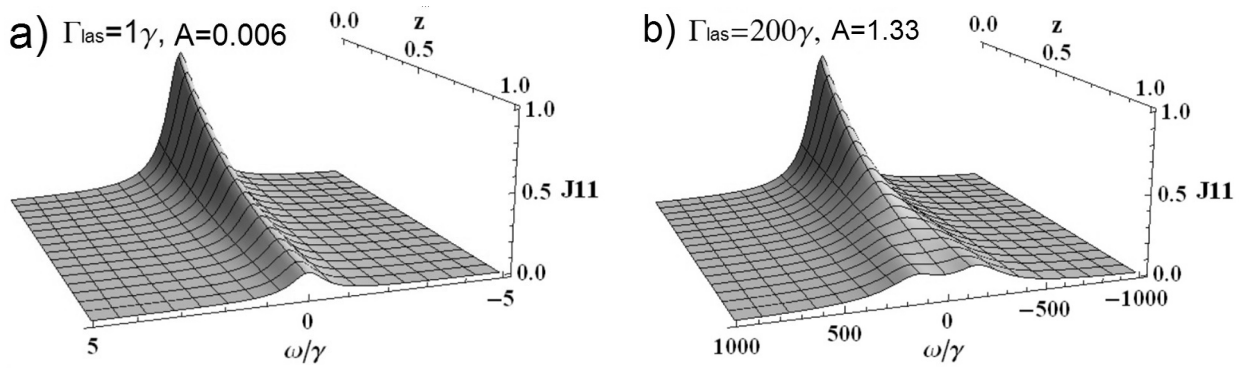

Fig. 3. The absorption of the frequency component $\omega_{1}$ of an energy spectrum when the emission passes through the medium in the case of a narrow (a) and wide (b) spectrum outside the CPT resonance (two-photon detuning $\delta=570 \mathrm{~Hz}$ ). When the laser bandwidth changes, the integral intensity of the emission stays permanent. The parameters are the same as in Fig. 2.

there is a high emission absorption outside the CPT resonance. Such absorption peculiarities result in spectrum distortions in the form of a gap (Fig. 3(b)).

When building a quantum frequency standard on the basis of the CPT effect one can use two different ways to detect the signal. One of such ways to record the signal is the analysis of the emission that has passed through the cell (Fig. 2(a)). The second way is to analyze the luminescence of the working atoms, i.e. the case when the photo detector registers the emission not in the direction of the laser ray. In the situation when the luminescence of the signal is analyzed, the power of that signal $P$ is proportional to the population of the top level of working atoms $\bar{\rho}_{33}(\delta)$ averaged over the cell length:

$$
P(\delta)=\hbar \omega \gamma^{\prime} n_{a} V \bar{\rho}_{33}(\delta),
$$

where $\omega$ is the frequency of optical transition, $\gamma^{\prime}$ is the rate of desintegration of the top level, $n_{a}$ is the concentration of working atoms, $\mathrm{V}$ is the volume of the cell. In Fig. 2(b) the contrast of the CPT resonance is shown according to the luminescence signal.

It is possible to evaluate the stability of the quantum standard frequency with the help of the quality parameter $\mathrm{Q}$ of the resonance, which is proportional to the ratio of the signal amplitude to the width of this signal at half-height. Then, the higher is Q, the greater is the frequency standard stability. Figure 4 shows the dependence of Q on the laser bandwidth. Looking at the graph, it is evident that, when the laser bandwidth increases, the quality parameter reduces for the input intensity signal (the solid curve). Mainly, this happens owing to the "tails" rising in Fig. 2(a) and the resulting fall in the resonance amplitude. The growth of $\mathrm{Q}$ for the luminescence signal is determined by other reasons, unlike the reduction in the quality for the intensity signal. Namely, if the laser bandwidth is narrow $(A<1$, the solid, dashed and dash-and-dot curves in Fig. 2(b)), all of the emission intensity is experienced by the atoms from a rather narrow velocity group. In this case a particular atom "captured" by the spectrum is effectively exposed to a greater intensity than in the situation with a broader spectrum $(A \geq 1$, the dotted and dash-and-dot with 


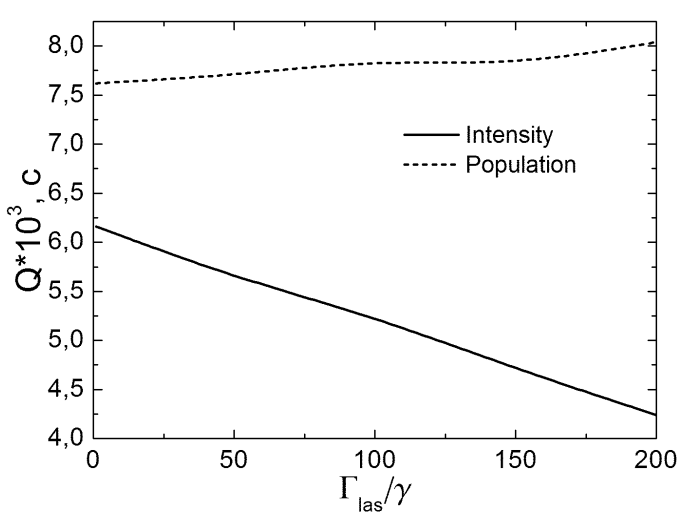

Fig. 4. The parameter of the CPT resonance quality depending on the laser bandwidth when measuring the transmitted intensity (the solid curve) and according to the luminescence signal (the dashed curve). When the bandwidth changes, the integral emission intensity remains constant. The parameters are the same as in Fig. 2.

two dots curves in Fig. 2(b)). It is known that the CPT resonance broadens when the intensity of the excitation fields rises - the so-called light broadening: $\Delta_{0} \approx$ $\nu^{g g^{\prime}}+\Omega^{2} / \gamma^{\prime}$, where $\Delta_{0}$ is the CPT resonance width, $\Omega^{2} \sim I$ - the square of the Rabi frequency is proportional to the intensity. ${ }^{10}$ Thus, when $\Gamma_{\text {las }}$ increases, the resonance width declines. Figure 2(b) makes it clear that the resonance amplitude falls simultaneously. Nevertheless, the ratio of the amplitude to width Q increases, but much weaker than for the input intensity signal.

\section{Conclusions}

The paper presents a theory of forming a CPT resonance for the finite laser bandwidth in an optically dense medium of $\Lambda$-atoms in a buffer gas cell. Within the framework of the developed theory the main equations for the density matrix are established together with the equations of the emission spectrum transfer via the medium.

The shape of the CPT resonance has been found when detecting the signal of the laser emission intensity which has been transmitted trough the cell, as well as the luminescence signal of the atoms. It has been shown that, if the laser bandwidth is "broad" $(A>1)$, the signal quality parameter of the transmitted intensity falls due to selective absorption and the distortion of the signal spectrum, and the quality parameter of the luminescence signal increases as a result of a decrease in the light broadening. On the contrary, if a laser with a "narrow spectrum" of emission is used $(A<1)$, the quality parameter of the transmitted intensity signal rises, as the spectrum is absorbed homogeneously, and the quality parameter of the luminescence signal drops due to the light broadening of the CPT resonance for the velocity group of atoms "captured" by the laser. 


\section{Acknowledgments}

This work was supported by State Assignment No. 2014/184, State Assignment No. 3.1446.2014K, Russian Foundation for Basic Research (projects 14-02-31422, 15-02-01013, 13-02-00944).

\section{References}

1. B. D. Agapiev, M. B. Gorniy, B. G. Matisov and Yu. V. Rozhdestvensky, UFN 163, 1 (1993).

2. M. B. Gorniy, B. G. Matisov and Yu. V. Rozhdestvensky, JETP 68, 728 (1989).

3. S. Harris, Physics Today 50, 36 (1997).

4. J. Vanier, Appl. Phys. 81, 421 (2005).

5. M. Stahler, R. Wynands, S. Knappe et al., Opt. Lett. 27, 1472 (2002).

6. A. Akulshin, A. Celikov and V. Velichansky, Opt. Commun. 84, 139 (1991).

7. D. Peter, D. Schwindt, S. Knappe et al., Appl. Phys. Lett. 85, 6409 (2004).

8. V. V. Yashuk, J. Granwehr, D. F. Kimbal et al., Phys. Rev. Lett. 93, 160801 (2004).

9. M. D. Lukin, Reviews of Modern Physics 75, 457 (2003).

10. M. Fleischhauer, A. Imamoglu and J. P. Marangos, Reviews of Modern Physics 77, 633 (2005).

11. G. Kazakov, B. Matisov, I. Mazets et al., Phys. Rev. A 72, 063408 (2005).

12. S. A. Zibrov, V. L. Velichanskiy, A. S. Zibrov et al., JETP Lett. 82, 534 (2005).

13. E. Breschi, G. Kazakov, R. Lammegger et al., Phys. Rev. A 79, 063837 (2009).

14. S. A. Zibrov, I. Novikova, D. F. Phillips et al., Phys. Rev. A 81, 013833 (2010).

15. X. Liu, J. Mérolla, S. Guérandel et al., Phys. Rev. A 87, 029903 (2013).

16. O. Firstenberg, M. Shuker, A. Ben-Kish et al., Phys. Rev. A 76, 013818 (2007).

17. R. H. Dicke, Phys. Rev. 89, 472 (1953).

18. I. E. Mazets and B.G. Matisov, JETP 26, 013818 (1992).

19. G. A. Kazakov, B. G. Matisov, I. E. Mazets et al., Phys. Rev. A 72, 063408 (2005).

20. G. A. Kazakov, B. G. Matisov, I. E. Mazets et al., JTP 76, 20 (2006).

21. S. G. Rautian, G. I. Smirnov and A. M. Shalagin, Nonlinear Sesonances in Atomic and Molecular Spectra (Nauka, Novosibirsk, 1979).

22. O. A. Kocharivskaya and Ya. I. Hanin, JETP 90, 1610 (1986).

23. A. G. Chirkov and B. G. Matisov, Modern Theory of Precision Generators Stability (SPbSTU Publishing House, St. Petersburg, 1979), p.76. 